PHYSICAL REVIEW D 90, 054018 (2014)

\title{
Diffusion of $\Lambda_{c}$ in hot hadronic medium and its impact on $\Lambda_{c} / D$ ratio
}

\author{
Sabyasachi Ghosh, ${ }^{1}$ Santosh K. Das, ${ }^{2,3}$ Vincenzo Greco, ${ }^{2,3}$ Sourav Sarkar, ${ }^{4}$ and Jan-e Alam ${ }^{4}$ \\ ${ }^{1}$ Instituto de Fsica Terica, Universidade Estadual Paulista, Rua Dr. Bento Teobaldo Ferraz, 271 Bloco II, \\ 01140-070 Sao Paulo, SP, Brazil \\ ${ }^{2}$ Department of Physics and Astronomy, University of Catania, Via S. Sofia 64, 1-95125 Catania, Italy \\ ${ }^{3}$ Laboratori Nazionali del Sud, INFN-LNS, Via S. Sofia 62, I-95123 Catania, Italy \\ ${ }^{4}$ Theoretical Physics Division, Variable Energy Cyclotron Centre, \\ I/AF, Bidhan Nagar, Kolkata 700064, India
}

(Received 24 July 2014; published 17 September 2014)

\begin{abstract}
The drag and diffusion coefficients of the $\Lambda_{c}(2286 \mathrm{MeV})$ have been evaluated in the hadronic medium which is expected to be formed in the later stages of the evolving fireball produced in heavy ion collisions at RHIC and LHC energies. The interactions between the $\Lambda_{c}$ and the hadrons in the medium have been derived from an effective hadronic Lagrangian, as well as from the scattering lengths obtained in the framework of heavy baryon chiral perturbation theory $(\mathrm{HB} \chi \mathrm{PT})$. In both the approaches, the magnitude of the transport coefficients turn out to be significant. A larger value is obtained in the former approach with respect to the latter. Significant values of the coefficients indicate a substantial amount of interaction of the $\Lambda_{c}$ with the hadronic thermal bath. Furthermore, the transport coefficients of the $\Lambda_{c}$ are found to be different from the transport coefficients of the $D$ meson. The present study indicates that the hadronic medium has a significant impact on the $\Lambda_{c} / D$ ratio in heavy ion collisions.
\end{abstract}

PACS numbers: 12.38.Mh, 24.85.+p, 25.75.-q, 25.75.Nq

\section{INTRODUCTION}

One of the primary aims of the ongoing nuclear collision programs at the Relativistic Heavy Ion Collider (RHIC) and Large Hadron Collider (LHC) energies is to create a new state of matter known as quark gluon plasma (QGP), the bulk properties of which are governed by the light quarks and gluons. Heavy quarks (HQs $\equiv$ charm and beauty) play crucial roles in understanding the properties of QGP [1], because they can witness the entire space-time evolution of the system as they are produced in the initial hard collision and remain extant during the evolution. Heavy flavor as a probe of the medium has generated significant interest in the recent past due to the suppression of its momentum distribution at large momentum in the thermal medium, denoted by $R_{A A}\left(p_{T}\right)$ [2-5] and its elliptic flow $\left(v_{2}\right)$ [4]. Several attempts have been made to study these factors within the framework of the Fokker-Plank equation [1,6-18] and Boltzmann equation [19-22]. However, the roles of hadronic phase have been ignored in these works.

In heavy ion collision (HIC) at ultrarelativistic energies, the appearance of the hadronic phase is inevitable. To make reliable characterization of the QGP, the role of the hadronic phase should be assessed and its contribution must be subtracted out from the data. Recently the diffusion coefficients of the $D$ and $B$ mesons have been evaluated in the hadronic phase [23-30] and their effects on $R_{A A}\left(p_{T}\right)$ at large transverse momentum $\left(p_{T}\right)$ [31] and elliptic flow $\left(v_{2}\right)[32,33]$ have been studied and found to be significant. Apart from the heavy mesons $(D$ and $B)$ the heavy baryon $\left(\Lambda_{c}\right)$ is also significant as its enhancement $[34,35]$ due to quark coalescence would affect the $R_{A A}\left(p_{T}\right)$ of nonphotonic electrons. Furthermore, the baryon-to-meson ratio, $\left(\Lambda_{c} / D\right)$, is fundamental for the understanding of inmedium hadronization [36] with respect to the light quark sector [37]. Enhancement of the heavy baryon-to-meson ratio $\left(\Lambda_{c} / D\right)$ in $\mathrm{Au}+\mathrm{Au}$ collisions compared to $\mathrm{p}+\mathrm{p}$ collisions affects the nonphotonic electron spectrum $\left(R_{A A}\right)$ [38-41]. The branching ratio for the process $\Lambda_{c} \rightarrow e+$ $X(4.5 \% \pm 1.7 \%)$ is smaller than $D \rightarrow e+X(17.2 \% \pm$ $1.9 \%)$, resulting in fewer electrons from decays of $\Lambda_{c}$ than $D$. Hence, enhancement of the $\Lambda_{c} / D$ ratio in $\mathrm{Au}+\mathrm{Au}$ collision will reduce the observed nonphotonic electrons. We notice that the $p_{T}$ dependence of the $\Lambda_{c} / D$ ratio may get modified further in the hadronic medium as the interactions of $\Lambda_{c}$ and $D$ with hadrons are non-negligible. Keeping this in mind, we attempt to study the transport coefficients (drag and diffusion coefficients) of $\Lambda_{c}$ in hadronic phase.

The paper is organized as follows. In the next section we discuss the formalism used to evaluate the drag and diffusion coefficients of the heavy flavored baryon in a hot hadronic matter. Section III is devoted to presenting the results. Section IV contains summary and discussions.

\section{FORMALISM}

The drag and diffusion coefficients of the charmed baryon $\Lambda_{c}$, propagating through a hot hadronic medium have been evaluated using the scattering length obtained in Ref. [42], where Liu et al. have estimated next-to-next-toleading order (NNLO) amplitudes in the framework of heavy baryon chiral perturbation theory $(\mathrm{HB} \chi \mathrm{PT})$. We consider the elastic interaction of $\Lambda_{c}$ with thermal pions, 
TABLE I. Table showing the extracted values of the $T$ matrix from the scattering length, $a$, which were obtained by Liu et al. [42].

\begin{tabular}{lcc}
\hline \hline$\Lambda_{c} M$ & $a(\mathrm{fm})$ & $T$ \\
\hline$\Lambda_{c} \pi$ & 0.06 & 9.28 \\
$\Lambda_{c} K$ & $-0.032 \pm 0.038$ & -12.42 to 1.06 \\
$\Lambda_{c} \bar{K}$ & $(0.79+0.27 \mathrm{i}) \pm 0.044$ & $(72.75+47.9 \mathrm{i})$ to $(207.6+47.9 \mathrm{i})$ \\
$\Lambda_{c} \eta$ & $(0.35+0.19 \mathrm{i}) \pm 0.044$ & $(55.27+34.32 \mathrm{i})$ to $(71.17+34.32 \mathrm{i})$ \\
\hline \hline
\end{tabular}

kaons, and $\eta$ mesons. The temperature of the bath can vary from $T_{c}(\sim 170)$ to $T_{F}(\sim 120 \mathrm{MeV})$, relevant for heavy ion collisions at RHIC and LHC energies. Here $T_{c}$ is the transition temperature at which the QGP formed in HIC makes a transition to hadrons and $T_{F}$ is the freeze-out temperature at which the hadrons cease to interact. In this temperature range the abundance of $\Lambda_{c}$ and $D$ is small and their thermal production and annihilation can be ignored. Hence, in evaluating the drag and diffusion coefficients of the $\Lambda_{c}$ only elastic processes will be considered.

For the elastic scattering of $\Lambda_{c}$ of momentum $p_{1}$ with a thermal hadron, $H$ of momentum $p_{2}$, i.e., for the process $\Lambda_{c}\left(p_{1}\right)+H\left(p_{2}\right) \rightarrow \Lambda_{c}\left(p_{3}\right)+H\left(p_{4}\right)$, the drag coefficient $\gamma$ can be expressed as [43] (see also $[44,45]$ )

$$
\gamma=p_{i} A_{i} / p^{2}
$$

where $A_{i}$ takes the form

$$
\begin{aligned}
A_{i}= & \frac{1}{2 E_{p_{1}}} \int \frac{d^{3} p_{2}}{(2 \pi)^{3} E_{p_{2}}} \int \frac{d^{3} p_{3}}{(2 \pi)^{3} E_{p_{3}}} \int \frac{d^{3} p_{4}}{(2 \pi)^{3} E_{p_{4}}} \\
& \times \frac{1}{g_{\Lambda_{c}}} \sum \overline{|\mathcal{M}|^{2}}(2 \pi)^{4} \delta^{4}\left(p_{1}+p_{2}-p_{3}-p_{4}\right) \\
& \times f\left(p_{2}\right)\left\{1 \pm f\left(p_{4}\right)\right\} \equiv\left\langle\left\langle\left(p_{1}-p_{3}\right)\right\rangle\right\rangle,
\end{aligned}
$$

where $g_{\Lambda_{c}}$ denotes the statistical degeneracy of $\Lambda_{c}$, and $f\left(p_{2}\right)$ is the Bose-Einstein (BE) or Fermi-Dirac (FD) distribution function depending upon the spin of $H$ in the initial channel. Similarly, the factor $1 \pm f\left(p_{4}\right)$ represents Bose enhanced or Pauli suppressed probability of the corresponding $H$ in the final channel. $|\bar{M}|^{2}$ represents the modulus square of the spin averaged matrix element for the $\Lambda_{c}+H$ elastic scattering process. Equation (2) illustrates that the drag coefficient is the measure of the thermal average of the momentum transfer, $p_{1}-p_{3}$, weighted by the interaction through $\overline{|\mathcal{M}|^{2}}$.

Similarly the diffusion coefficient $B$ can be expressed as

$$
\left.B=\frac{1}{4}\left[\left\langle p_{3}^{2}\right\rangle\right\rangle-\frac{\left\langle\left\langle\left(p_{1} \cdot p_{3}\right)^{2}\right\rangle\right\rangle}{p_{1}^{2}}\right] .
$$

With an appropriate choice of $T\left(p_{3}\right)$, both the drag and diffusion coefficients can be expressed in a single equation as follows:

$$
\begin{aligned}
\ll T\left(p_{1}\right) \gg & =\frac{1}{512 \pi^{4}} \frac{1}{E_{p_{1}}} \int_{0}^{\infty} \frac{p_{2}^{2} d p_{2} d(\cos \chi)}{E_{p_{2}}} \\
& \times \hat{f}\left(p_{2}\right)\left\{1 \pm f\left(p_{4}\right)\right\} \frac{\lambda^{\frac{1}{2}}\left(s, m_{p_{1}}^{2}, m_{p_{2}}^{2}\right)}{\sqrt{s}} \\
& \times \int_{1}^{-1} d\left(\cos \theta_{\text {c.m. }}\right) \frac{1}{g} \sum \overline{|\mathcal{M}|^{2}} \int_{0}^{2 \pi} d \phi_{\text {c.m. }} T\left(p_{3}\right),
\end{aligned}
$$

where $\lambda\left(s, m_{p_{1}}^{2}, m_{p_{2}}^{2}\right)=\left\{s-\left(m_{p_{1}}+m_{p_{2}}\right)^{2}\right\}\left\{s-\left(m_{p_{1}}-m_{p_{2}}\right)^{2}\right\}$ is the triangular function.

The drag and diffusion coefficients of $\Lambda_{c}$ can be evaluated in hadronic matter by using $\overline{|T|^{2}}$ [27] in place of $\overline{|\mathcal{M}|^{2}}$ in Eq. (4), where the momentum independent $T$-matrix elements simply estimate the strength of the $\Lambda_{c}$ interactions with the thermal hadrons.

The scattering lengths of $\Lambda_{c}$ with light pseudoscalar mesons $M=\pi, K, \bar{K}$, and $\eta$ have recently been obtained by Liu et al. [42] in the framework of $\mathrm{HB} \chi \mathrm{PT}$. From the scattering lengths, $a$ (say) of $\Lambda_{c}$ interacting with $M$, we can extract the $T$-matrix element by using the relation

$$
T=4 \pi\left(m_{\Lambda_{c}}+m_{M}\right) a,
$$

where $m_{\Lambda_{c}}$ and $m_{M}$ are the masses of $\Lambda_{c}$ and mesons $(M)$, respectively. From the scattering lengths ( $a$ in $\mathrm{fm}$ ), the extracted values of $T$ are given in Table I.

In Ref. [42] Liu et al. have fixed the low energy constants (LECs) with the help of relations based on quark model symmetry, heavy quark spin symmetry, $S U(4)$ flavor symmetry, and some empirical relations. However, a dimensionless constant $\alpha^{\prime}$ remains unknown, which is taken in the natural range $[-1,1][42,46]$. Therefore, the table shows a band of numerical values of $T$ matrices, which are corrected up to the third order $\left[\mathcal{O}\left(\epsilon^{3}\right)\right]$ with the explicit power counting in $\mathrm{HB} \chi \mathrm{PT}$. Using the $T$ matrices from Table I and corresponding $\mathrm{BE}$ distributions for $H=M=\pi, K, \eta$ in Eq. (4), we can get an estimate of drag and diffusion coefficients of $\Lambda_{c}$ in hadronic matter.

Besides the scattering length approach, we have also investigated the contributions of the drag and diffusion coefficients, resulting from the Born-like scattering: $\Lambda_{c} \pi \rightarrow \Sigma_{c} \rightarrow \Lambda_{c} \pi$. Using the effective hadronic Lagrangian [47], 
DIFFUSION OF $\Lambda_{c}$ IN HOT HADRONIC $\ldots$

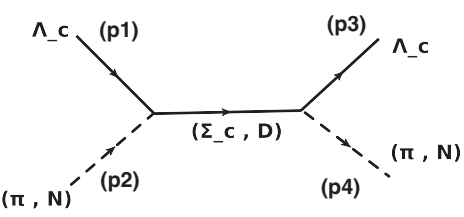

(A)

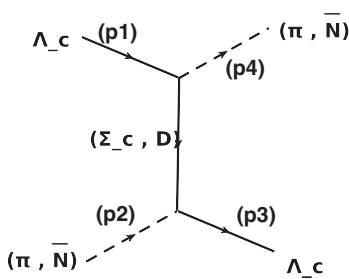

(B)
FIG. 1. Feynman diagrams for the scattering of $\Lambda_{c}$ with the pion, nucleon, and antinucleon in the medium.

$$
\mathcal{L}_{\Lambda_{c} \Sigma_{c} \pi}=\frac{g}{m_{\pi}} \bar{\Lambda}_{c} \gamma^{5} \gamma^{\mu} \operatorname{Tr}\left(\vec{\tau} \cdot \overrightarrow{\Sigma_{c}} \vec{\tau} \cdot \vec{\pi}\right)+\text { H.c. },
$$

we can calculate the matrix elements for $\Lambda_{c} \pi$ scattering diagrams via $\Sigma_{c}$. The Lagrangian is based on the gauged $S U(4)$ flavor symmetry but with empirical masses. The coupling constant $g=0.37$ is taken from Ref. [47], where $S U(4)$ relations are used to fix it.

The possible $s$ and $u$ channel diagrams for $\Lambda_{c}+\pi \rightarrow$ $\Sigma_{c} \rightarrow \Lambda_{c}+\pi$ processes are shown in the panels (a) and (b) of Fig. 1. The matrix elements for the two channels are, respectively, given by

$M_{s}^{\Lambda_{c} \pi}=-\left(\frac{2 g}{m_{\pi}}\right)^{2}\left[\bar{u}\left(p_{3}\right) \gamma^{5} \not p_{4} \frac{\left(\not p_{1}+\not p_{2}+m_{\Sigma_{c}}\right)}{\left(s-m_{\Sigma_{c}}^{2}\right)} \gamma^{5} \not p_{2} u\left(p_{1}\right)\right]$

and

$M_{u}^{\Lambda_{c} \pi}=-\left(\frac{2 g}{m_{\pi}}\right)^{2}\left[\bar{u}\left(p_{3}\right) \gamma^{5} \not p_{2} \frac{\left(\not p_{1}-\not p_{4}+m_{\Sigma_{c}}\right)}{\left(u-m_{\Sigma_{c}}^{2}\right)} \gamma^{5} \not p_{4} u\left(p_{1}\right)\right]$.

Similarly from the Lagrangian density [47],

$$
\mathcal{L}_{\Lambda_{c} N D}=\frac{f}{m_{D}} \bar{N} \gamma^{5} \gamma^{\mu} \Lambda_{c} \partial_{\mu} D+\partial_{\mu} \bar{D} \overline{\Lambda_{c}} \gamma^{5} \gamma^{\mu} N,
$$

one can obtain the matrix elements for the processes $\Lambda_{c} N \rightarrow D \rightarrow \Lambda_{c} N$ and $\Lambda_{c} \bar{N} \rightarrow D \rightarrow \Lambda_{c} \bar{N}$ (see Fig. 1). The modulus square of the spin averaged total amplitudes $\overline{|\mathcal{M}|^{2}}$ for all processes are given in the Appendix. Using those $\overline{|\mathcal{M}|^{2}}$ from the effective hadronic model as well as the corresponding BE and FD distributions for $H=\pi$ and $N$ in Eq. (4), we can get alternative estimates of the drag and diffusion coefficients of $\Lambda_{c}$ in the hadronic medium. We have included form factors in each of the interaction vertices to take into account the finite size of the hadrons. For the $u$ and $s$ channel diagrams the form factors are taken as [47] $F_{u}=\Lambda^{2} /\left(\Lambda^{2}+\vec{q}^{2}\right)$ and $F_{s}=\Lambda^{2} /$ $\left(\Lambda^{2}+{\overrightarrow{p_{i}}}^{2}\right)$, respectively, where $\vec{q}$ is the three-momentum transfer, $p_{i}$ is the initial momentum of the pions, and $\Lambda=1 \mathrm{GeV}$.
PHYSICAL REVIEW D 90, 054018 (2014)

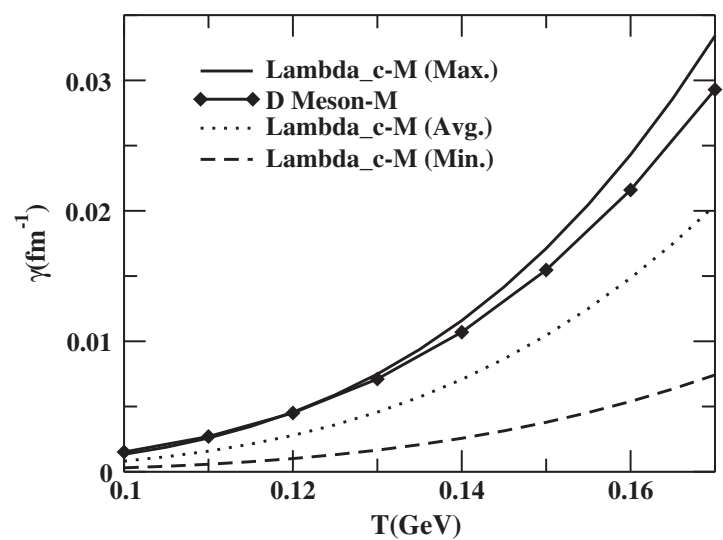

FIG. 2. Variation of the drag coefficient with temperature for the $\Lambda_{c}$ and $D$ meson [27] in a mesonic medium, where their interaction strengths are governed from their scattering lengths (SL) [42].

\section{RESULTS AND DISCUSSION}

Let us first discuss the results of the drag coefficients obtained from the $T$-matrix elements of $\Lambda_{c} M$ scattering, given in Table I. The variation of the drag coefficient of $\Lambda_{c}$ with temperature is depicted in Fig. 2 and compared with the drag coefficient of the $D$ mesons [27] while propagating through the same thermal medium consisting of pions, kaons, and eta. The magnitude of the drag coefficients is quite significant, indicating a substantial interaction of $\Lambda_{c}$ with the thermal hadrons. The maximum and minimum values of the drag coefficient for $\Lambda_{c}$ correspond to the band associated with the $T$-matrix element presented in Table I. The average value of the drag of $\Lambda_{c}$ is found to be smaller than that of $D$.

The single electron spectrum originating from the decays of $\Lambda_{c}$ and $D$ measured in HIC is sensitive to the following two mechanisms: (i) the production of $\Lambda_{c}$ in HIC is enhanced compared to that in $\mathrm{pp}$ because of the direct interaction of $c$ with $[u d]$ bound states available in the QGP [34], (ii) the $\Lambda_{c}$ has a smaller branching ratio to semileptonic decay than $D$. These two mechanisms lead to a deficiency of electrons at intermediate $p_{T}$ $\left[2<p_{T}(\mathrm{GeV})<5\right]$ [38]. If the drag of $\Lambda_{c}$ is more (less) than $D$, then that will further reduce (enhance) the electrons in this domain of $p_{T}$. We find here that the value of the drag of $\Lambda_{c}$ has a band of uncertainties as shown in Fig. 2; therefore, it is not possible to draw a conclusion regarding which way the drag of $\Lambda_{c}$ will contribute to the electron spectra originating from the decays of charm mesons and baryons. However, measurements of $D$ meson spectra via hadronic and semileptonic channels in the same collision conditions will help in estimating the electron spectra from $\Lambda_{c}$ and hence its drag coefficients.

For a hadronic system of lifetime, $\Delta \tau$, and drag $\gamma$, the momentum suppression is approximately given by $R_{A A} \sim$ $e^{-\Delta \tau \gamma}$ [24]. Picking up a value of $\gamma$ of $D$ at $T=170 \mathrm{MeV}$ 


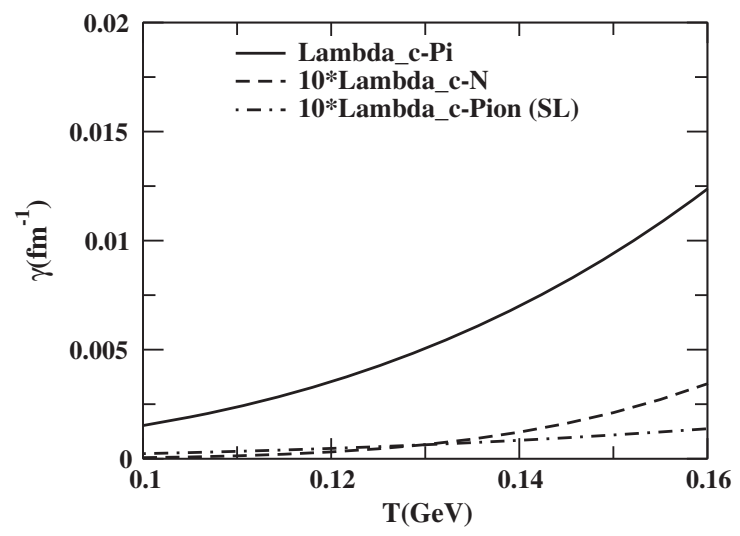

FIG. 3. Variation of the drag coefficient with temperature for $\Lambda_{c}$ in a pionic medium, using the dynamics of effective Lagrangian (EL) and scattering length (SL). The contribution of the drag coefficient for $\Lambda_{c} N$ scattering in the EL dynamics is also presented.

from the results displayed in Fig. 2, we get $R_{A A} \sim 14 \%$ for $\Delta \tau=5 \mathrm{fm} / \mathrm{c}$. The values of $R_{A A}$ for $\Lambda_{c}$ at the same temperature and $\Delta \tau$ are about $16 \%$ and $4 \%$, respectively, for maximum and minimum values of $\gamma$ shown in Fig. 2. Similarly the $\Lambda_{c} / D$ ratio at the same temperature and $\Delta \tau$ is approximately given by $\Lambda_{c} / D \sim e^{\Delta \tau\left(\gamma_{D}-\gamma_{\Lambda_{c}}\right)}$, where $\gamma_{D}$ and $\gamma_{\Lambda_{c}}$ are the drag coefficients of the $D$ meson and $\Lambda_{c}$, respectively. The $\Lambda_{c} / D$ ratio can vary up to $12 \%$ depending on the minimum to maximum value of the drag coefficients of $\Lambda_{c}$.

The temperature variation of the drag coefficient of $\Lambda_{c}$ in a pionic medium has been depicted in Fig. 3. Here EL corresponds to the matrix element obtained from the effective hadronic Lagrangian [47] and SL corresponds to the scattering length or the $T$-matrix element obtained from the $\mathrm{HB} \chi \mathrm{PT}$. We found that the drag of $\Lambda_{c}$ in pionic medium for EL is much larger than that for SL.

The corresponding SL results for the momentum diffusion coefficient as a function of temperature are depicted in Fig. 4. The difference between the maximum and minimum values of both coefficients gets larger at higher temperature as displayed in Figs. 2 and 4.

The variation of the ratio of $\Lambda_{c}$ to $D$ is shown in Fig. 5 as a function of $p_{T}$. The Fokker-Planck (FP) equation has been used to study the time evolution of the $D$ and $\Lambda_{c}$ in the hadronic bath of the equilibrated degrees of freedom. This is given by $[31,43]$

$$
\frac{\partial f}{\partial t}=\frac{\partial}{\partial p_{i}}\left[A_{i}(p) f+\frac{\partial}{\partial p_{j}}\left[B_{i j}(p) f\right]\right]
$$

where $f$ is the momentum distribution of the nonequilibrated degrees of freedom, and $A_{i}(p)$ and $B_{i j}(p)$ are related to the drag and diffusion coefficients. The interaction between the probe and the thermal bath enter through the drag and diffusion coefficients. The initial distributions

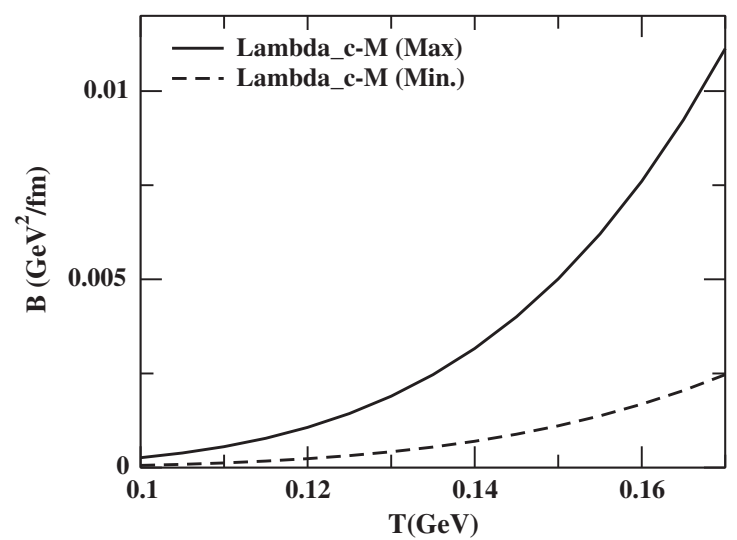

FIG. 4. Variation of the diffusion coefficient with temperature, when $\Lambda_{c}$ interacts with all the light pseudoscalar mesons $M$ (in SL dynamics).

of the $D$ meson and $\Lambda_{c}$ are obtained (at the end of QGP phase) by using the fragmentation and coalescence techniques of [36] and results of [8]. Their ratio (at the end of the QGP phase) has been shown in the solid line of Fig. 5. In the present calculation of $\Lambda_{c}$ spectra, resonances are not taken into account at variance with Ref. [35].

The ratio estimated after evolving the $D$ [31] and $\Lambda_{c}$ in the hadronic medium through the Fokker-Planck equation is displayed in Fig. 5. In Fig. 5 QGP refers to the ratio at the end of the QGP phase and "QGP + hadronic" refers to the ratio at the end of the hadronic phase. Maximum and minimum of the ratio correspond to the maximum and minimum values of the drag and diffusion coefficients of $\Lambda_{c}$. The results indicate that the ratio gets enhanced for $2 \leq p_{T} \leq 7$ due to the interactions of the $D$ and $\Lambda_{c}$ while propagating through the hadronic medium. Such enhancement will have interesting consequences on the nuclear suppression of the charm quarks in QGP measured through the single electron spectra originating from the decays of charmed hadrons.

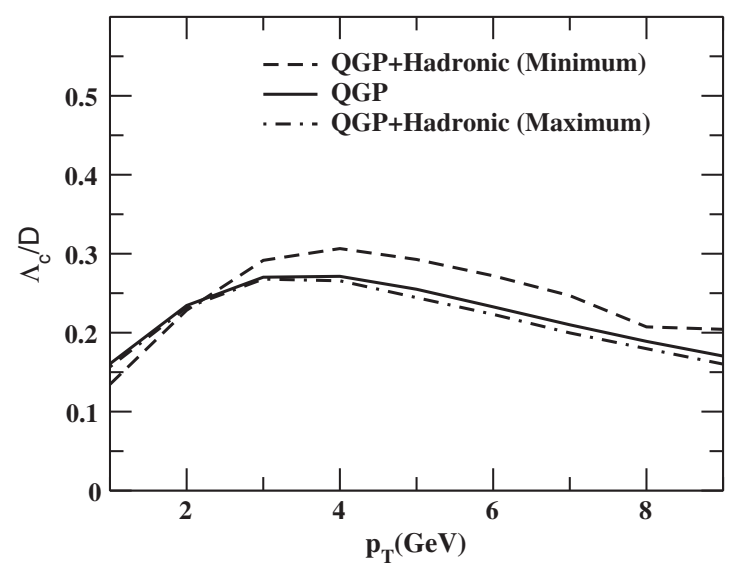

FIG. 5. Transverse momentum variation of the $\Lambda_{c}$ to $D$ ratio has been displayed for maximum and minimum values of the drag of $\Lambda_{c}$ (see text). 


\section{SUMMARY AND DISCUSSION}

We have studied the diffusion of $\Lambda_{c}$ in a hot hadronic medium. Using scattering amplitudes, obtained by Liu et al. [42] in the framework of $\mathrm{HB} \chi \mathrm{PT}$, we have evaluated the drag and diffusion coefficients of the $\Lambda_{c}$ interacting with a hadronic background composed of pions, kaons, and eta. We have also calculated the drag coefficients of the $\Lambda_{c}$ interacting with the pion and nucleon, using an effective hadronic Lagrangian. It is found that the coefficients in the pionic medium, obtained from the effective hadronic Lagrangian, are quite higher than those obtained from the dynamics of scattering length. However, the coefficients resulting from the $\Lambda_{c} N$ scattering obtained within an effective hadronic model approach are comparable to the coefficients estimated in the scattering length approach. The value obtained for the $\Lambda_{c}$ has been compared with the drag coefficient of the $D$ meson calculated within the framework of heavy meson chiral perturbation theory. It is found that the value of the drag coefficient of $\Lambda_{c}$ is generally lower than that of $D$ mesons. This result shows a significant effect on the $p_{T}$ dependence of the $\Lambda_{c} / D$ ratio and hence also on $R_{A A}$ of single electrons originating from the decay of $\Lambda_{c}$.

\section{ACKNOWLEDGMENTS}

S. G. is thankful to Z.W. Liu and S. L. Zhu for their useful suggestions via email. S. G. is supported by FAPESP, Grant No. 2012/16766-0. S. K. D. and V. G. acknowledge the support by the ERC StG under the QGPDyn Grant No. 259684.

\section{APPENDIX}

The modulus square of the spin averaged total amplitude for the processes of $\Lambda_{c}+\pi \rightarrow \Sigma_{c} \rightarrow \Lambda_{c}+\pi$ is given by

$$
\begin{aligned}
\overline{\left|M^{\Lambda_{c} \pi}\right|^{2}}= & \frac{3}{2}\left(\frac{2 g}{m_{\pi}}\right)^{4}\left[\frac{A_{s s}}{\left(s-m_{\Sigma_{c}}^{2}\right)^{2}}+\frac{A_{u u}}{\left(u-m_{\Sigma_{c}}^{2}\right)^{2}}\right. \\
& \left.+\frac{2 A_{s u}}{\left(s-m_{\Sigma_{c}}^{2}\right)\left(u-m_{\Sigma_{c}}^{2}\right)}\right],
\end{aligned}
$$

where

$$
\begin{aligned}
A_{s s}= & {\left[-2 m_{\pi}^{2} m_{\Lambda_{c}}\left(s-m_{\Lambda_{c}}^{2}\right)^{2}\left(m_{\Lambda_{c}}+2 m_{\Sigma_{c}}\right)\right.} \\
& +m_{\pi}^{4}\left(s+m_{\Lambda_{c}}^{2}+2 m_{\Lambda_{c}} m_{\Sigma_{c}}\right)^{2} \\
& \left.-\left(s-m_{\Lambda_{c}}^{2}\right)^{2}\left(s u-m_{\Lambda_{c}}^{4}+t m_{\Sigma_{c}}^{2}\right)\right], \\
A_{u u}= & {\left[-2 m_{\pi}^{2} m_{\Lambda_{c}}\left(u-m_{\Lambda_{c}}^{2}\right)^{2}\left(m_{\Lambda_{c}}+2 m_{\Sigma_{c}}\right)\right.} \\
& +m_{\pi}^{4}\left(u+m_{\Lambda_{c}}^{2}+2 m_{\Lambda_{c}} m_{\Sigma_{c}}\right)^{2} \\
& \left.-\left(u-m_{\Lambda_{c}}^{2}\right)^{2}\left(s u-m_{\Lambda_{c}}^{4}+t m_{\Sigma_{c}}^{2}\right)\right],
\end{aligned}
$$

and

$$
\begin{aligned}
A_{s u}= & {\left[-4 m_{\pi}^{6} m_{\Lambda_{c}}^{2}+(4 s-2 t+4 u) m_{\Lambda_{c}}^{6}-2 m_{\Lambda_{c}}^{8}\right.} \\
& +2 t\left(s^{2}-t^{2}-2 s u+u^{2}\right) m_{\Lambda_{c}} m_{\Sigma_{c}}+8 t^{2} m_{\Lambda_{c}}^{3} m_{\Sigma_{c}} \\
& +m_{\Lambda_{c}}^{4}\left\{-3 s^{2}+5 t^{2}+2 s(t-3 u)+2 t u-3 u^{2}-2 t m_{\Sigma_{c}}^{2}\right\} \\
& -\left(s^{2}-t^{2}+u^{2}\right)\left(s u-t m_{\Sigma_{c}}^{2}\right)+m_{\Lambda_{c}}^{2}\left\{s^{3}+6 t^{2} m_{\Sigma_{c}}^{2}\right. \\
& \left.-(t-u)(t+u)^{2}+s^{2}(t+3 u)-s\left(t^{2}+4 t u-3 u^{2}\right)\right\} \\
& +2 m_{\pi}^{4}\left\{s u+3 m_{\Lambda_{c}}^{4}-4 t m_{\Lambda_{c}} m_{\Sigma_{c}}+8 m_{\Lambda_{c}}^{3} m_{\Sigma_{c}}\right. \\
& \left.-2 t m_{\Sigma_{c}}^{2}-m_{\Lambda_{c}}^{2}\left(t+4 m_{\Sigma_{c}}^{2}\right)\right\}-2 m_{\pi}^{2}\left\{m _ { \Lambda _ { c } } m _ { \Sigma _ { c } } \left\{s^{2}\right.\right. \\
& \left.-4 t^{2}+s(t-2 u)+t u+u^{2}\right\}+14 t m_{\Lambda_{c}}^{3} m_{\Sigma_{c}} \\
& +8 m_{\Lambda_{c}}^{4}\left(t+m_{\Sigma_{c}}^{2}\right)+2 t\left(s u-t m_{\Sigma_{c}}^{2}\right)+m_{\Lambda_{c}}^{2}\left\{s^{2}-2 t^{2}\right. \\
& \left.\left.\left.-t u+u^{2}-s(t+2 u)+(-4 s+6 t-4 u) m_{\Sigma_{c}}^{2}\right\}\right\}\right] .
\end{aligned}
$$

The modulus square of the spin averaged total amplitude for the processes of $\Lambda_{c}+N \rightarrow D \rightarrow \Lambda_{c}+N$ and $\Lambda_{c}+$ $\bar{N} \rightarrow D \rightarrow \Lambda_{c}+\bar{N}$ are, respectively, given by

$$
\begin{aligned}
\overline{\left|M^{\Lambda_{c} N}\right|^{2}}= & \frac{1}{2}\left(\frac{f}{m_{D}}\right)^{4} \frac{1}{\left(s-m_{D}^{2}\right)^{2}} \\
& \times \operatorname{Tr}\left[\left(p_{3}+m_{\Lambda_{c}}\right)\left(p_{1}+\not p_{2}\right)\left(p_{4}-m_{N}\right)\left(\not p_{1}+p_{2}\right)\right] \\
& \times \operatorname{Tr}\left[\left(p_{2}-m_{N}\right)\left(p_{1}+p_{2}\right)\left(p_{1}+m_{\Lambda_{c}}\right)\left(p_{1}+\not p_{2}\right)\right] \\
= & \frac{2\left(f / m_{D}\right)^{4}}{\left(s-m_{D}^{2}\right)^{2}}\left(m_{\Lambda_{c}}-m_{N}\right)^{2}\left\{s-\left(m_{\Lambda_{c}}+m_{N}\right)^{2}\right\} \\
& \times\left[3\left(m_{\Lambda_{c}}^{4}+m_{N}^{4}\right)+10 m_{\Lambda_{c}}^{2} m_{N}^{2}+(t+u)^{2}-s^{2}\right. \\
& \left.-4(t+u)\left(m_{\Lambda_{c}}^{2}+m_{N}^{2}\right)+s\left(m_{\Lambda_{c}}-m_{N}\right)^{2}\right]
\end{aligned}
$$

and

$$
\begin{aligned}
\overline{\left|M^{\Lambda_{c} \bar{N}}\right|^{2}}= & \frac{1}{2}\left(\frac{f}{m_{D}}\right)^{4} \frac{1}{\left(u-m_{D}^{2}\right)^{2}} \\
& \times \operatorname{Tr}\left[\left(\not p_{3}+m_{\Lambda_{c}}\right)\left(\not p_{1}-p_{4}\right)\left(p_{2}+m_{N}\right)\left(\not p_{1}-\not p_{4}\right)\right] \\
& \times \operatorname{Tr}\left[\left(p_{1}+m_{\Lambda_{c}}\right)\left(\not p_{1}-p_{4}\right)\left(\not p_{4}+m_{N}\right)\left(\not p_{1}-\not p_{4}\right)\right] \\
= & \frac{2\left(f / m_{D}\right)^{4}}{\left(u-m_{D}^{2}\right)^{2}}\left(m_{\Lambda_{c}}-m_{N}\right)^{2}\left\{u-\left(m_{\Lambda_{c}}+m_{N}\right)^{2}\right\} \\
& \times\left[3\left(m_{\Lambda_{c}}^{4}+m_{N}^{4}\right)+10 m_{\Lambda_{c}}^{2} m_{N}^{2}+(t+s)^{2}-u^{2}\right. \\
& \left.-4(t+s)\left(m_{\Lambda_{c}}^{2}+m_{N}^{2}\right)+u\left(m_{\Lambda_{c}}-m_{N}\right)^{2}\right] .
\end{aligned}
$$


[1] R. Rapp and H. van Hees, Quark Gluon Plasma 4, edited by R. C. Hwa and X. N. Wang (World Scientific, Singapore, 2010), p. 111.

[2] B. I. Abeleb et al. (STAR Collaboration), Phys. Rev. Lett. 98, 192301 (2007).

[3] A. Adare et al. (PHENIX Collaboration), Phys. Rev. Lett. 98, 172301 (2007).

[4] S. S. Adler et al. (PHENIX Collaboration), Phys. Rev. Lett. 96, 032301 (2006).

[5] B. Abeleb et al. (ALICE Collaboration), J. High Energy Phys. 09 (2012) 112.

[6] G. D. Moore and D. Teaney, Phys. Rev. C 71, 064904 (2005).

[7] H. van Hees, V. Greco, and R. Rapp, Phys. Rev. C 73, 034913 (2006).

[8] H. van Hees, M. Mannearelli, V. Greco, and R. Rapp, Phys. Rev. Lett. 100, 192301 (2008).

[9] Y. Akamatsu, T. Hatsuda, and T. Hirano, Phys. Rev. C 79, 054907 (2009).

[10] C. M. Ko and W. Liu, Nucl. Phys. A783, 233 (2007).

[11] S. K. Das, J. Alam, and P. Mohanty, Phys. Rev. C 80, 054916 (2009); 82, 014908 (2010); S. Majumdar, T. Bhattacharyya, J. Alam, and S. K. Das, Phys. Rev. C 84, 044901 (2011).

[12] W. M. Alberico, A. Beraudo, A. De Pace, A. Molinari, M. Monteno, M. Nardi, and F. Prino, Eur. Phys. J. C 71, 1666 (2011); W. M. Alberico, A. Beraudo, A. De Pace, A. Molinari, M. Monteno, M. Nardi, F. Prino, and M. Sitta, ibid. 73, 2481 (2013).

[13] C. Young, B. Schenke, S. Jeon, and C. Gale, Phys. Rev. C 86, 034905 (2012).

[14] S. Cao and S. A. Bass, Phys. Rev. C 84, 064902 (2011).

[15] M. He, R. J. Fries, and R. Rapp, Phys. Rev. Lett. 110, 112301 (2013).

[16] S. K. Das and A. Davody, Phys. Rev. C 89, 054912 (2014).

[17] T. Lang, H. van Hees, J. Steinheimer, and M. Bleicher, arXiv:1208.1643.

[18] H. Xu, X. Dong, L. Ruan, Q. Wang, Z. Xu, and Y. Zhang, Phys. Rev. C 89, 024905 (2014).

[19] P. B. Gossiaux and J. Aichelin, Phys. Rev. C 78, 014904 (2008).

[20] J. Uphoff, O. Fochler, Z. Xu, and C. Greiner, Phys. Rev. C 84, 024908 (2011).

[21] M. Younus, C. E. Coleman-Smith, S. A. Bass, and D. K. Srivastava, arXiv:1309.1276.

[22] S. K. Das, F. Scadina, and V. Greco, arXiv:1312.6857.
[23] M. Laine, J. High Energy Phys. 04 (2011) 124.

[24] M. He, R. J. Fries, and R. Rapp, Phys. Lett. B 701, 445 (2011).

[25] S. Ghosh, S. K. Das, S. Sarkar, and J. Alam, Phys. Rev. D 84, 011503 (2011).

[26] L. Abreu, D. Cabrera, F. J. Llanes-Estrada, and J. M. Torres-Rincon, Ann. Phys. (Amsterdam) 326, 2737 (2011).

[27] S. K. Das, S. Ghosh, S. Sarkar, and J. Alam, Phys. Rev. D 85, 074017 (2012).

[28] L. Abreu, D. Cabrera, and J. M. Torres-Rincon, Phys. Rev. D 87, 034019 (2013).

[29] L. Tolos and J. M. Torres-Rincon, Phys. Rev. D 88, 074019 (2013).

[30] J. M. Torres-Rincon, L. Tolos, and O. Romanets, Phys. Rev. D 89, 074042 (2014).

[31] S. K. Das, S. Ghosh, S. Sarkar, and J. Alam, Phys. Rev. D 88, 017501 (2013).

[32] M. He, R. J. Fries, and R. Rapp, arXiv:1401.3817.

[33] V. Ozvenchuk, J. M. TorresRincon, P. B. Gossiaux, L. Tolos and J. Aichelin, arXiv:1408.4938.

[34] S. H. Lee, K. Ohnishi, S. Yasui, I. Yoo, and C. M. Ko, Phys. Rev. Lett. 100, 222301 (2008).

[35] Y. Oh, C. M. Ko, S. H. Lee, and S. Yasui, Phys. Rev. C 79, 044905 (2009).

[36] V. Greco, R. Rapp, and C. M. Ko, Phys. Lett. B 595, 202 (2004).

[37] V. Greco, C. M. Ko, and P. Levai, Phys. Rev. Lett. 90, 202302 (2003); Phys. Rev. C 68, 034904 (2003).

[38] P. Sorensen and X. Dong, Phys. Rev. C 74, 024902 (2006).

[39] G. Martinez-Garcia, S. Gadrat, and P. Crochet, Phys. Lett. B 663, 55 (2008).

[40] A. Ayala, J. Magnin, L. M. Montano, and G. T. Sanchez, Phys. Rev. C 80, 064905 (2009).

[41] R. J. Fries, V. Greco, and P. Sorensen, Annu. Rev. Nucl. Part. Sci. 58, 177 (2008)

[42] Z. W. Liu and S. L. Zhu, Phys. Rev. D 86, 034009 (2012); Nucl. Phys. A914, 494 (2013).

[43] B. Svetitsky, Phys. Rev. D 37, 2484 (1988).

[44] M. G. Mustafa, D. Pal, and D. K. Srivastava, Phys. Rev. C 57, 889 (1998).

[45] S. K. Das, V. Chandra, and J. Alam, J. Phys. G 41, 015102 (2014).

[46] F. K. Guo, C. Hanhart, and U. G. Meissner, Eur. Phys. J. A 40, 171 (2009).

[47] W. Liu and C. M. Ko, Phys. Lett. B 533, 259 (2002). 